\title{
New lightweight construction prospects enabled by hydroforming
}

\author{
Markus Werner ${ }^{a}$, Verena Psyk, André Albert, Marco Pröhl, Petr Kurka, Dirk Landgrebe, \\ and Welf-Guntram Drossel \\ Fraunhofer Institute for Machine Tools and Forming Technology, Reichenhainer Strasse 88, \\ 09126 Chemnitz, Germany
}

\begin{abstract}
To reduce weight of a component and improve its performance, the part properties have to be adapted to the requirements, the load profile, and the function of the product. This leads to a continuously increasing complexity of the components necessitating innovative manufacturing strategies for producing the desired shapes from materials that are difficult to handle. Hydroforming offers high potential for fulfilling this demand, especially, if it is applied in deliberate process integrations, combinations, and chains that allow exploiting complementary advantages of all technologies involved. The paper presents four examples of such manufacturing strategies: magnetic pulse welding followed by tube hydroforming, deep drawing combined with injection moulding and hydroforming using the molten plastic, free bending or roll forming followed by hydroforming, and deep drawing with integrated media forming.
\end{abstract}

\section{Introduction and motivation}

For economical, ecological, and sometimes even ergonomical reasons, industry is obliged to produce lighter, more performant, and cheaper products. For a long time, the automotive industry has been being a driving force in developing lightweight design strategies that are both: economically affordable and suitable for mass production. During the last years, other industrial sectors such as the energy sector have been intensifying research and development in lightweight designs, too.

A decrease of the component weight and an improvement of its performance will be enabled if the material and geometry of the part are ideally adapted to the requirements, the load profile, and the function of the product. This was clearly shown by scientific research [1] and successful industrial case studies [2]. However, such an adaptation of the part properties leads to a continuously increasing complexity of the components. Therefore, innovative manufacturing processes are required. These technologies must be capable of producing the desired shapes from materials and material systems that are difficult to handle, such as aluminium and magnesium alloys, high strength steels, (fibre-reinforced) plastic, or even multi-material systems [3]. Hydroforming technologies offer high potential for fulfilling this demand and the capability of these processes can even be increased if they are applied in deliberate

\footnotetext{
${ }^{\text {a }}$ Corresponding author: markus . werner@iwu.fraunhofer.de
}

This is an Open Access article distributed under the terms of the Creative Commons Attribution License 4.0, which permits unrestricted use, distribution, and reproduction in any medium, provided the original work is properly cited. 
a)

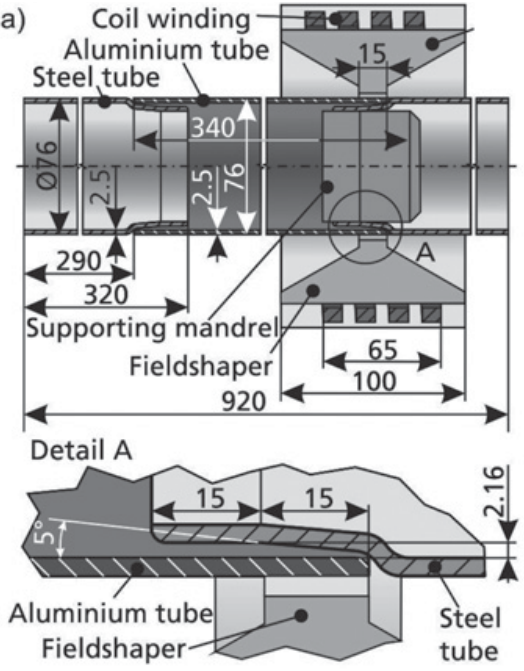

b)
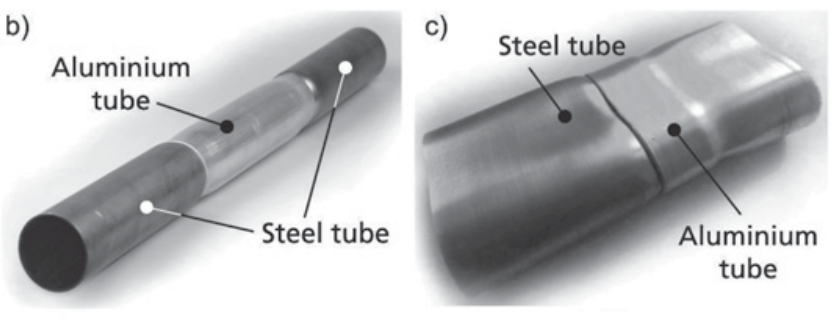

d)

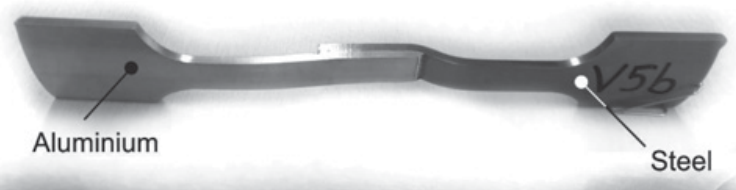

e)

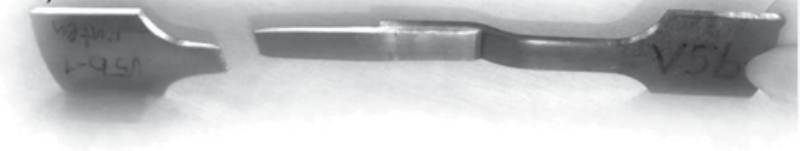

Figure 1. (a) Setup for magnetic pulse welding; (b) magnetic pulse welded hybrid tube; (c) joint section after severe deformation of the cross section; (d) specimen for lap shear tests prepared from the joint section before testing; (e) specimen for lap shear tests prepared from the joint section after testing.

process integrations, process combinations, and process chains that allow exploiting complementary advantages of all technologies involved. At the Fraunhofer Institute for Machine Tools and Forming Technology (IWU) the invention, analysis, and development of such process combinations has been a research focus for several years. Recently, the focus was put especially on:

- magnetic pulse welding followed by tube hydroforming,

- deep drawing combined with injection moulding and hydroforming using the molten plastic,

- free bending or roll forming followed by hydroforming, and

- deep drawing with integrated media forming.

\section{Magnetic pulse welding followed by tube hydroforming}

Magnetic pulse welding is a high speed process using pulsed magnetic fields to join similar or dissimilar tube or sheet metal materials without largely heating the parts and thus preventing disadvantages of conventional (i.e. thermal) welding methods, such as heat distortion, forming of intermetallic phases, softening of the joining region, etc. [4]. In the regarded process combination, magnetic pulse welding is used for producing hybrid aluminium steel tubes as tailored semi-finished parts for hydroforming. Here, both sides of an aluminium tube are connected to steel tubes featuring a tapered end that enables positioning in the aluminium tube with some overlap as shown in Fig. 1(a). The resulting hybrid tube allows significant weight reduction compared to the conventional full steel solution and keeping the required steel properties, where it is necessary at the same time.

For the analysis and design of the process combination a combined numerical and experimental approach was applied. In the first step, the requirement profile that has to be satisfied by the hybrid tube and specifically by the joints was characterised: During hydroforming, the hybrid tube has to endure significant deformation of the cross section without leakage. A numerical simulation of the hydroforming process has shown that the joint has to withstand a maximum shear force of approximately $42 \mathrm{kN}$ [5]. Dividing this value by the length of the weld seam $(209.5 \mathrm{~mm})$ leads to a mean required force per length of $200 \mathrm{~N} / \mathrm{mm}$ for the aluminium-steel-joint. 
a) basic concept

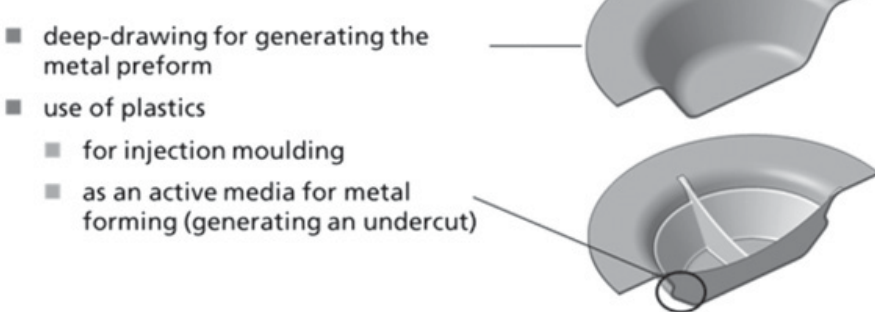

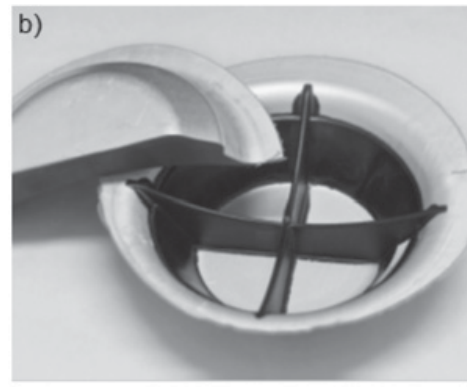

Figure 2. Combination of technologies: deep drawing, injection moulding, and forming using the molten plastic: a) process concept; b) realized demonstrator part.

In the next step an appropriate inductor system consisting of a suitable tool coil and a fieldshaper was designed for the magnetic pulse welding process (see Fig. 1a). For dimensioning this tool a methodology efficiently combining simplified decoupled and more accurate coupled electromagnetic and structural mechanical simulations was used $[6,7]$. The inductor system was realised by Poynting $\mathrm{GmbH}$ (Germany) and applied at IWU for producing hybrid tubes depicted in Fig. 1(b).

These were characterised in model experiments representing the major load cased during hydroforming: Lap shear tests performed on specimens prepared from the joint area as illustrated in Fig. 1(d) proved a maximum transferable force of $2.45 \mathrm{kN}$, corresponding to a force per length of the weld seam of $245 \mathrm{~N} / \mathrm{mm}$. This is clearly above the required value calculated by the numerical simulation above. As shown in Fig. $1 €$, failure in the lap shear test occurred far away from the joint. This proved that the strength of the joint is higher compared to the strength of the aluminium parent material. The cross section deformation tests conducted on the joint section is illustrated in Fig. 1(c). These tests proved that even after closing of the hydroforming tools, the hybrid tube was still watertight. Additional burst tests showed that the specimens fail due to bulging and subsequent cracking of the aluminium tube without leakage in the joint area. For the final feasibility proof of the suggested process combination of magnetic pulse welding and subsequent hydroforming, technological hydroforming tests are contemplated.

\section{Deep drawing combined with injection moulding and media based forming using the molten plastic}

In the state of the art production technologies for metal / plastic hybrid components, the metal parts are produced separately from the plastic parts [8]. The injection moulding process is only used for forming the plastic parts and for joining. These process chains are very extensive.

Within the framework of the Federal cluster of excellence MERGE, in cooperation of the Fraunhofer IWU and the TU Chemnitz, an innovative process combining the technologies of deep drawing, injection moulding, and forming by means of the molten plastic was designed. This combination of technologies enables manufacturing sheet based metal/plastic hybrid components in-situ in one die using only one process. One part can be produced with every single stroke of the press.

The manufacturing process designed consists of two stages. In the first step, the sheet to be formed is fed into the die and deep drawn. After deep drawing the plastic is injected. This process step serves both: the manufacturing of the plastic component and the media based forming of the metal component. Figure 2 shows the investigated test part: a cup with interior ribbing and undercut.

To make deep drawing feasible, the die was designed in such a manner that the process is run on a deep drawing press. A bolt on unit was applied to the injection moulding procedure. The die concept 

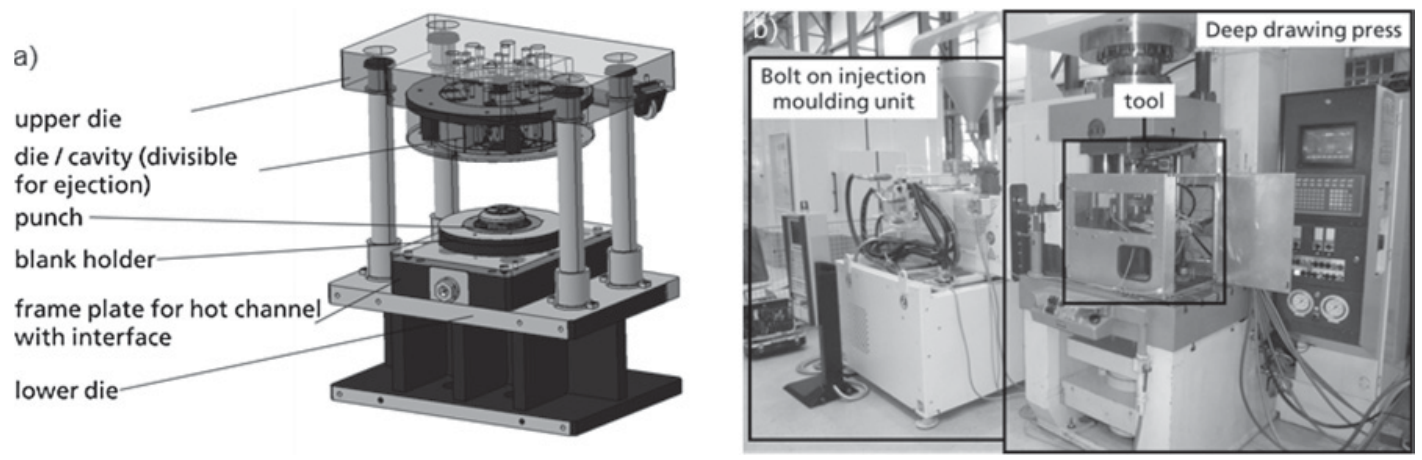

Figure 3. (a) Hybrid deep drawing and injection moulding die; (b) experimental setup for the process combination deep drawing, injection moulding and forming with the molten plastic.

and the experimental setup are shown in Fig. 3. The adhesion was not only achieved by the form fit that was enabled by the manufactured undercut, but also by the use of a chemical bonding agent.

\section{CNC bending or roll forming followed by hydroforming}

Blades of the currently common very large horizontal axis wind turbines are usually manually made of costly fibre reinforced materials [9]. The reason is their continuing size growth and the accordingly increasing importance of minimizing their weight. However, in recent years a second trend towards much smaller, urban compatible wind turbines, often with a vertical -not horizontal-rotation axis can be observed [10]. These turbines offer the freedom of rethinking the materials and manufacturing processes that are used for producing their blades. Here, metal materials and hydroforming techniques have a high cost and weight saving potential. Compared to fibre reinforced materials, the damage behaviour of metal under fatigue load conditions is much better predictable leading to significantly lower safety factors during design [11].

However, the circular shaped, tubular semi-finished products normally used for hydroforming processes are not reasonable for complex, aerodynamically shaped blade profiles. Instead, a process combination of $\mathrm{CNC}$ bending or roll forming a laser welded preform that is subsequently calibrated by hydroforming offers the possibility to build very stiff, closed profiles with a high degree of geometrical flexibility regarding the profile's cross sections [12]. Additionally, the two different preform processes ensure the profitability over a maximized range of quantities / piece numbers. An integrated reinforcement can further be added to the blade to increase its stiffness, resulting in a two chamber profile for sophisticated hydroforming processes, especially when considering the design of the sealing stamps for the profile's ends. The HyBlade project to manufacture small vertical axis wind turbine rotor was based on the principle, which integrates these manufacturing processes.

A small vertical axis wind turbine rotor shows that lightweight metal solutions are still competitive with regards to weight, durability and especially production cost compared to the turbine rotor manufactured from fibre reinforced materials. Within the project, beside the aerodynamic investigations, the structural effects of several design parameters like blade length and width, sheet thickness, and material have been investigated. Shown in Fig. 4(e) is the scaled down demonstrator designed and manufactured with combined processes. Depicted in Figs. 4(a)-(d) are the manufacturing processes combined namely: $\mathrm{CNC}$ bending, laser welding, and hydroforming to prove the feasibility of the process combination. Considering the positive results, a real size rotor with steel blades is built for aerodynamic tests on a commercial turbine at a certified wind test field. 

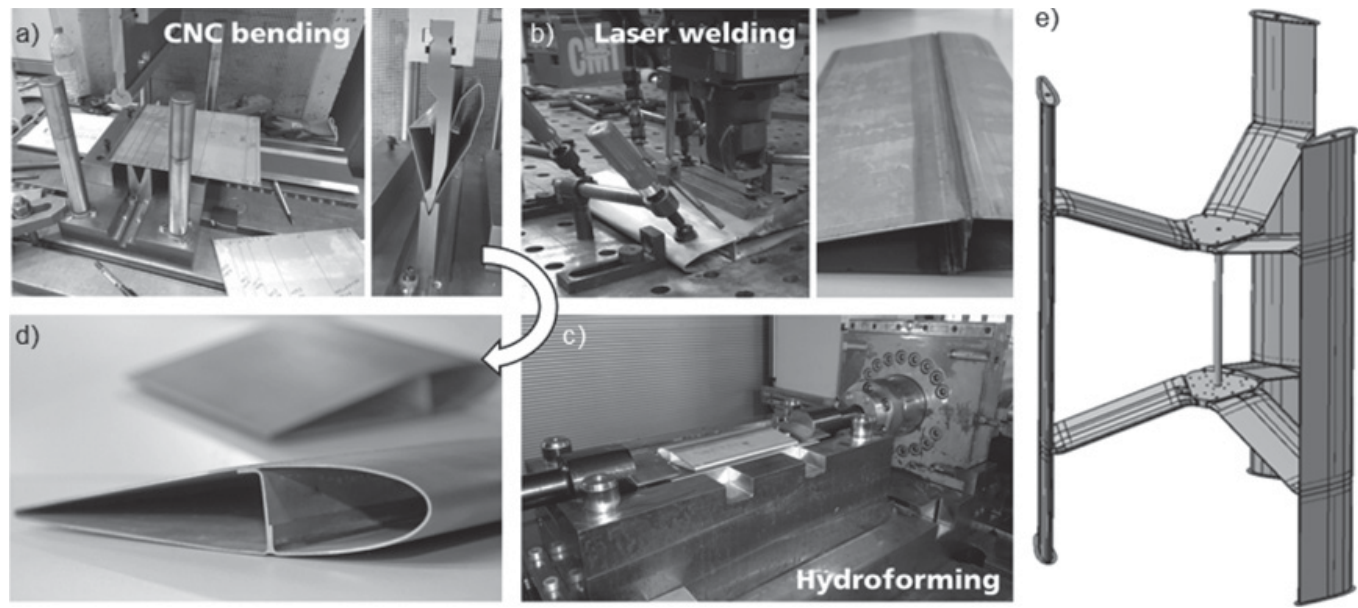

Figure 4. Process chain applying (a) CNC bending, (b) laser welding, and (c) hydroforming for the manufacturing of (d) aerodynamically shaped NACA0018 metal blades with an integrated reinforcement and (e) three blade metal rotor design for the vertical axis wind turbine.

Under operational conditions the centrifugal forces are dominant. Consequently, the load on a blade increases in the same way as its weight increases. Therefore, the ratio between density and Young's modulus (also known as "lightweight factor") is an important point when choosing the material. Since this ratio is nearly constant for metals and glass fibre reinforced plastics (GFRP) and just varies for the much more expensive carbon fibre pendant (CFRP), one can basically use the same sheet thickness for different materials and achieve a comparable stress and bending behaviour of the blade. The resulting weights for a $2.8 \mathrm{~m}$ high and $2.0 \mathrm{~m}$ wide rotor therefore decrease from $260 \mathrm{~kg}$ using steel to $95 \mathrm{~kg}$ using magnesium blanks. Figure 4(e) shows the rotor design of the vertical axis wind turbine. The realised steel blades prove that the hydroforming of such complex profiles is feasible and has a $90 \%$ cost reduction potential compared to the extensive manual processes for fibre reinforced parts.

\section{Deep drawing with integrated media forming}

In the last process combination presented here a conventional deep drawing process is augmented by an integrated local hydroforming step. Here, instead of a fluid medium an elastomer block enclosed by a steel frame is used for forming local details such as stiffening structures or design elements e.g. for part personalisation. The implementation of appropriate stiffening structures allows using a smaller wall thickness of the sheet and thus reducing weight while increasing the load capacitance [13]. The spectrum of possible structure geometries included regular patterns as shown in Fig. 5(a), but a requirement and load adapted structuring is much more likely irregular and shown in Fig. 5(b).

An important advantage of hydroforming compared to conventional deep drawing is the higher tool flexibility. According to [14] only one tool has to be shaped according to the desired workpiece geometry while the working media (here the elastomer punch) can be applied for forming different designs. Consequently, high cost saving potential is achievable especially with regards to product varieties. Compared to conventional hydroforming with fluid medium and an external pressure unit, forming with an elastomer punch does not require complex and expensive equipment, because the pressure is generated directly in the elastomer due to the punch movement. Moreover, cleaning and drying effort is reduced. 


\section{MATEC Web of Conferences}

a)

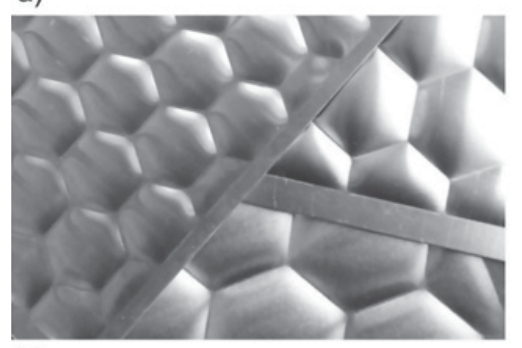

b)

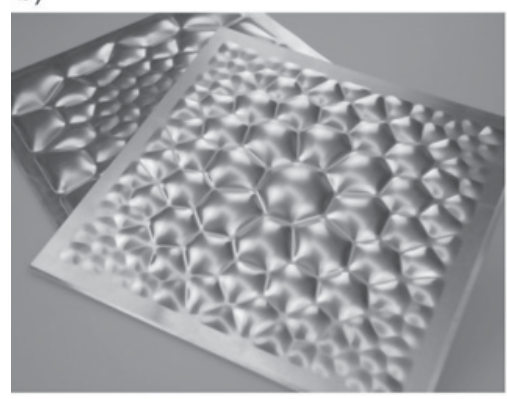

c)
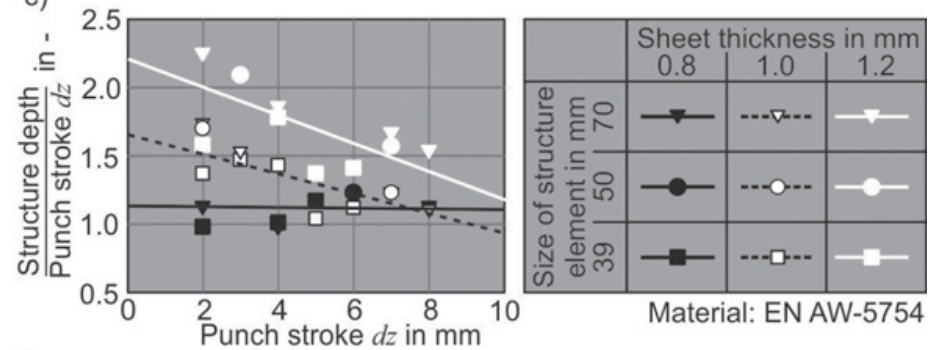

d)

Moment of first contact of sheet, Moment of maximum deformation die, and elastomer $t=t_{1}$

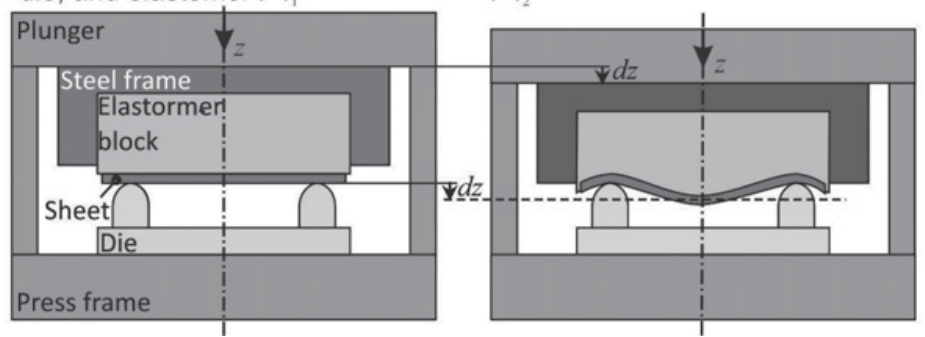

Figure 5. Examples of (a) regular and (b) irregular structures; (c) relation of punch stroke and resulting structure depth; (d) principle sketch of forming with elastomer tool.

However, considering the process design, there are some special issues, to be taken into consideration when forming with an elastomer tool. As an example the depth of the form element significantly depends on the punch stroke. As expected, it increases with increasing punch stroke (as long as it is not limited by a full die), but in contrast to experience gained in conventional forming techniques using solid tools, the depth can be significantly higher compared to the punch stroke as exemplarily shown for regular hexagonal comb structures of different sizes in Fig. 5®. This effect is especially pronounced in case of large structuring elements and small punch strokes.

The principle phenomenon can be attributed to the deformation of the elastomer as illustrated in the principle sketches in Fig. 5(d). During the process the plunger moves the elastomer punch towards the workpiece. At the moment of first contact of elastomer and workpiece $t_{1}$, the elastomer block still has its initial geometry and the sheet workpiece still is flat. In case of a further movement of the plunger $d z$ the further displacement of the elastomer and the workpiece is locally inhibited in the areas where workpiece and die are already in contact. This results in a local bulging of the elastomer in negative $z$ direction. Due to the incompressibility of the elastomer the total elastomer volume cannot change. This directly leads to a bulging in positive $z$-direction in those areas that are still free to deform. Consequently, the displacement of the elastomer and the workpiece is higher compared to the punch stroke, here. Depending on the size of the form element the resulting maximum dome hight of the form element can exceed the maximum punch stroke after the process and springback of the sheet. Based on preparing investigations of the forming with elastomer tools itself, future work will focus on the integration into a deep drawing process in order to allow local structuring of large sheet metal components.

The published research results were achieved within the following projects: "Skalierbare Module aus Antrieb und Achse für die Elektromobilität - ESKAM", funded by the Federal Ministry of Education and Research, "MERGE - Technologiefusion für multifunktionale Leichtbaustrukturen", funded within the frame of the Exzellenzinitiative des Bundes und der Länder, "Hydroformed blades for meshes of vertical axis wind turbines - HyBlade", funded by 
the Arbeitsgemeinschaft industrieller Forschungsvereinigungen - AiF (reg. 79EBR) within the European research program CORNET, and "Lokal versteifte Leichtbaukomponenten", funded by the Sächsische Aufbaubank SAB with financial means of the Europäischer Fonds für Regionale Entwicklung EFRE. The authors wish to thank for the financial support.

\section{References}

[1] M. Kleiner, S. Chatti, A. Klaus, J. Mat. Proc. Tech. 177, 2-7 (2006)

[2] AUDI AG (editor), Workshop Audi A3, (2012), https: //www . audi-mediaservices . com

[3] M. Hillebrecht, Karosseriebautage Hamburg, 13 ATZ Fachtagung, Hamburg, pp. 23-34 (2014)

[4] V. Psyk, D. Risch, B.L. Kinsey, A.E. Tekkaya, M. Kleiner, J. Mat. Proc. Tech. 211, 787-829 (2011)

[5] V. Psyk, T. Lieber, P. Kurka, W.-G. Drossel, Procedia CIRP 23, 1-6 (2014)

[6] V. Psyk, C. Scheffler, W.-G. Drossel, ZWF, 108, 831-8361 (2013)

[7] R. Neugebauer, V. Psyk, C. Scheffler, Adv. Mat. Res. 1018, 333-340 (2014)

[8] M. Geiger, G.W. Ehrenstein, Tagungsband zum Berichts- und Industriekolloquium des SFB 396, p.156 (2003)

[9] E. Hau, Wind Turbines - Fundamentals, Technologies, Application, Economics, Springer (2013)

[10] J. O. Dabiri, Journal of renewable and sustainable energy, 3, 043104 (2011)

[11] European Standard IEC 61400-2:2006, Wind turbines - Part 2: Design requirements for small wind turbines, Annex E: Partial Safety factors for Materials, Brussels (2006)

[12] D. Dominguez, M. Pröhl, T. Troyer, G. Feng, M. Werner, M. Runacres, Reducing blade manufacturing cost while maintaining aerodynamic performance of Vertical Axis Wind Turbines, EWEA Conference, Barcelona (2014)

[13] V. Psyk, P. Kurka, S. Kimme, M. Werner, D. Landgrebe, A. Ebert, M. Schwarzendahl, Optimisation of component performance via structuring, Proceedings of the $4^{\text {th }}$ Int. Conf. on New Forming Technology, Glasgow, UK, MATEC Web of Conferences, (2015)

[14] S.H. Zhang, Journal of Materials Processing Technology 151, 165-177 (2004) 